\title{
Normal and Injured Ankle Ligaments on Ultrasonography With Magnetic Resonance Imaging Correlation
}

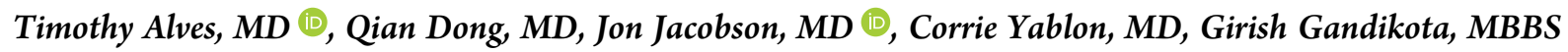

Received April 11, 2018, from the Department of Radiology, University of Michigan, Ann Arbor, Michigan USA. Manuscript accepted for publication May 22, 2018.

We thank Danielle Dobbs for medical illustrations.

Address correspondence to Qian Dong, MD, Department of Radiology, University of Michigan, 1500 E Medical Center Dr, TC 2904, Ann Arbor, MI 48109 USA.

E-mail: bingch@med.umich.edu

\begin{abstract}
Abbreviations
AITFL, anterior inferior tibiofibular ligament; ATFL, anterior talofibular ligament; CFL, calcaneofibular ligament; MRI, magnetic resonance imaging; PITFL, posterior inferior tibiofibular ligament; PTFL, posterior talofibular ligament; US, ultrasonography
\end{abstract}

doi:10.1002/jum.14716
Ultrasonography (US) has been increasingly used in the evaluation of ankle ligamentous injuries given its advantages as a dynamic, efficient, noninvasive, and cost-effective imaging method. Understanding the anatomy of the ankle ligaments is critical for correct diagnosis and treatment. This pictorial essay describes and illustrates the US scanning technique and potential pitfalls in evaluating the ankle ligaments and also provides an overview of the US appearance of normal and injured ankle ligaments with magnetic resonance imaging correlation. Highlighted structures include the lateral complex, medial/deltoid complex, spring (calcaneonavicular) ligament complex, and syndesmosis.

Key Words - ankle; ligaments; magnetic resonance imaging; musculoskeletal; ultrasonography

7 he ankle is the most commonly injured major joint in the body, with ankle ligamentous sprains being extremely common sports injuries. ${ }^{1}$ Imaging plays an important role as an adjunct to the physical examination in the evaluation of ligamentous injuries of the ankle. Although magnetic resonance imaging (MRI) is superior in evaluations of bone marrow and articular cartilage abnormalities, ultrasonography (US) has been found to be an effective method to evaluate the integrity of the ankle ligaments in particular. ${ }^{1-8}$ Ultrasonography provides the advantages of dynamic imaging, noninvasiveness, lower cost, and quicker scan times. It is also helpful in patients with contraindications to MRI or local metallic implants, which could create substantial artifacts. The main limitation of US in the evaluation of the ankle ligaments is operator depe'ndence, which can be overcome by a thorough understanding of the scanning technique and potential pitfalls, allowing the recognition of normal and injured US appearances of the ankle ligaments. The purpose of this pictorial essay is 2 -fold: (1) to review the US examination technique and scanning pitfalls in the evaluation of the ankle ligaments; and (2) to illustrate the US appearances of normal and injured ankle ligaments with MRI correlation. This study was approved by the Institutional Review Board at our institution and was performed in a Health Insurance Portability and Accountability Act-compliant manner.

\section{Ultrasonographic Technique and General Principles}

Ultrasonography of the ankle is typically completed with the patient supine and the foot and ankle on the examination table, 
and a high-frequency transducer of at least $10 \mathrm{MHz}$ should be used, preferably 15 to $18 \mathrm{MHz} .^{9}$ A small footprint, or "hockey stick," transducer can be used as needed depending on the ligament size and patient anatomy. The ankle ligaments are best evaluated in the long axis, with short-axis imaging for problem solving. Similar to other ligaments, the ankle ligaments should appear as echogenic with a more compact fibrillar structure than tendons, most commonly connecting two osseous structures. A general consideration regarding patient positioning is that the ligament should be slightly taut during evaluation to

Figure 1. Illustrations of the normal anatomy of the lateral complex: anterior view (A), posterior view (B), and lateral view (C). Black arrowheads indicate PITFL; stars, interosseous membrane; and white arrowheads, AITFL.
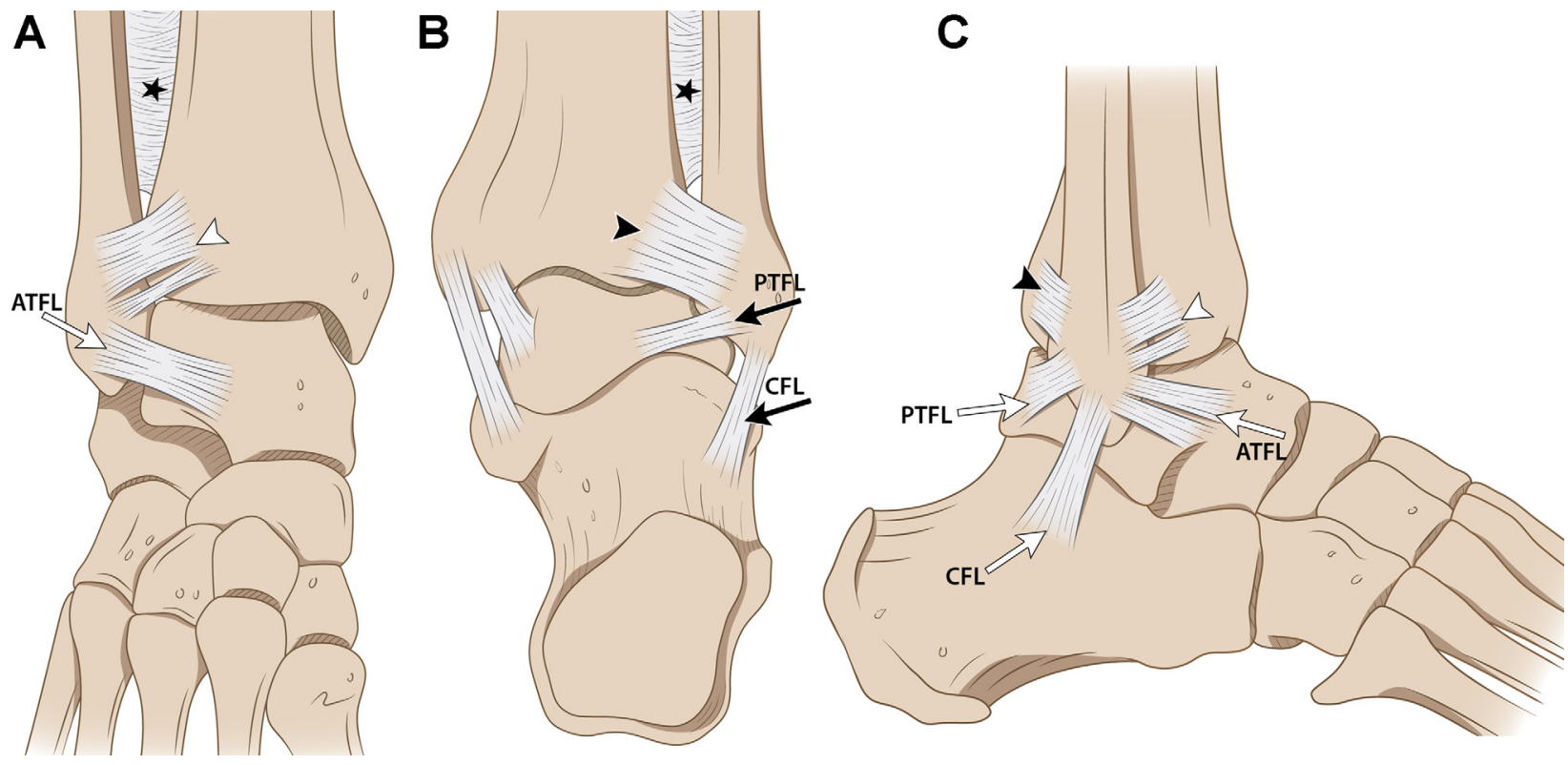

Figure 2. Ultrasonographic appearance of the ATFL. With the ankle slightly inverted, the transducer is placed parallel to the sole of the foot between the distal tip of the lateral malleolus (LM) and talus (A). The ATFL can be seen as a hyperechoic fibrillar band (arrowheads in B). Angling the transducer can help avoid an anisotropic appearance of the ligament (C). Corresponding T2-weighted MR image shows a normal ATFL (arrowhead in $\mathbf{D}$ ).
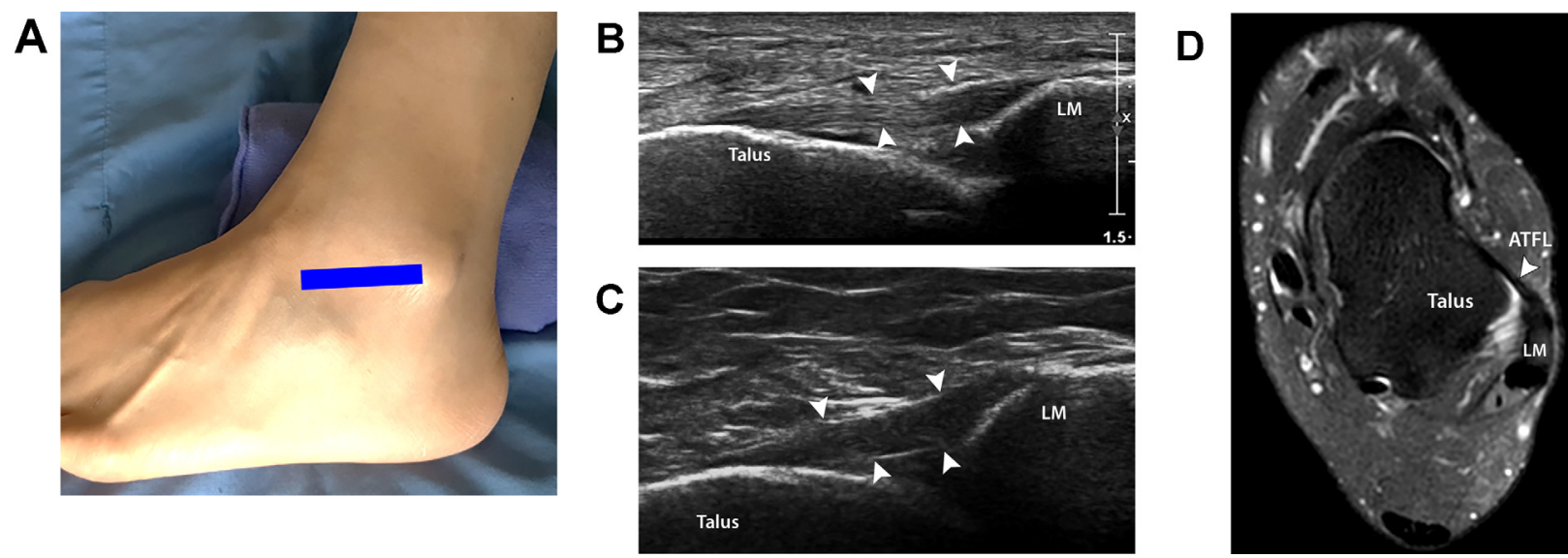
eliminate redundancy. The transducer should be angled so that the ligament fibers are perpendicular to the sound beam to eliminate anisotropy. Normal thickness of the ankle ligaments ranges from 2 to $5 \mathrm{~mm}^{8}$

Some general principles apply when it comes to the US appearance of ligament injuries. Acute partial- thickness ligament tears typically appear as hypoechoic thickening with preservation of some continuous fibers. ${ }^{2}$ Acute full-thickness ligament tears typically present as discontinuity, nonvisualization of the ligament, or visualization of hypoechoic or heterogeneous material representing a torn ligament and hemorrhage. ${ }^{2}$ Dynamic US may show lack of normal

Figure 3. Ultrasonographic appearance of the normal PTFL (A). Axial T2-weighted (B) and T1-weighted (c) MR images show normal marked signal heterogeneity and thickening (arrowheads) caused by the presence of fat striations, which should not be misinterpreted as a tear. LM indicates lateral malleolus; and star in $\mathbf{A}$, peroneal tendons.
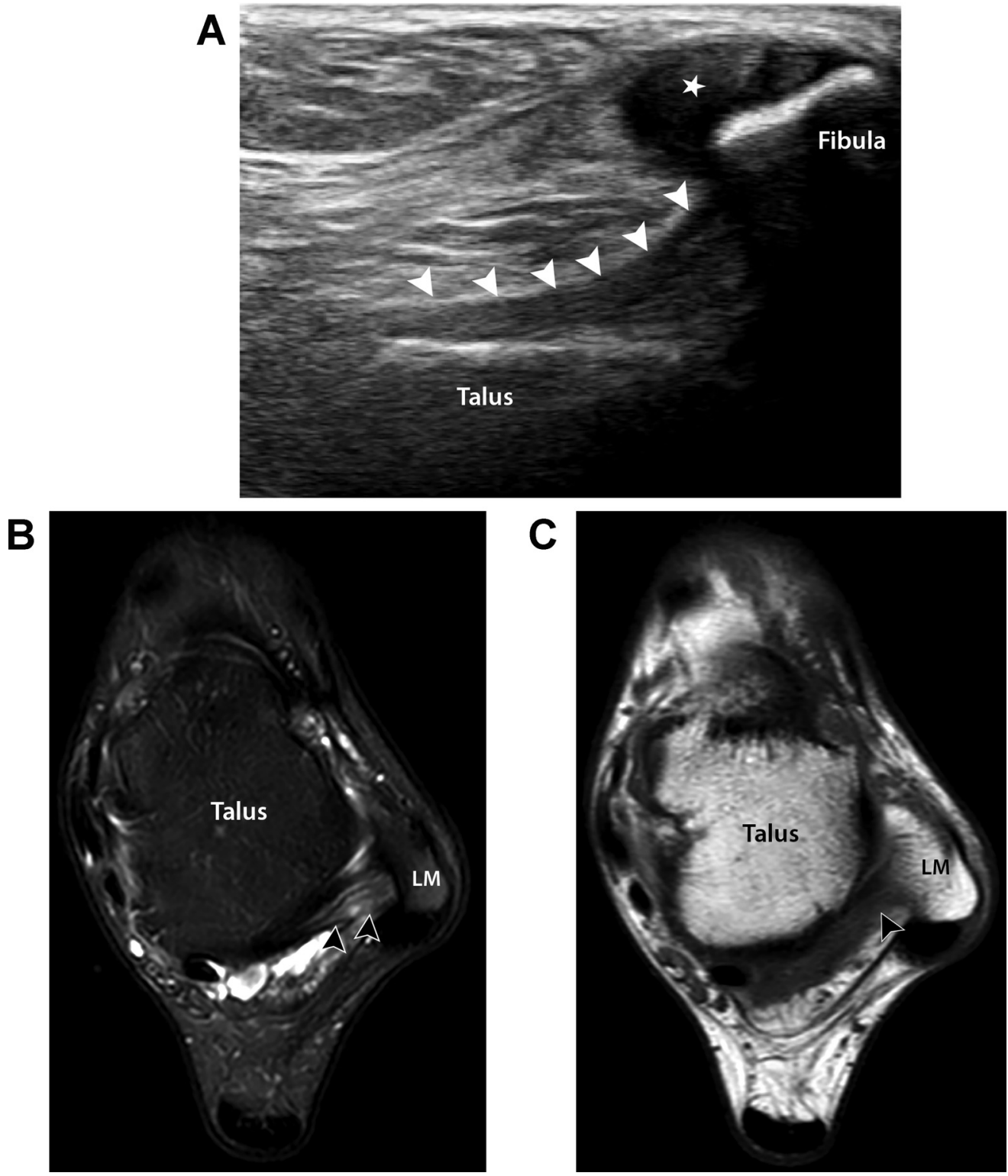
tendon tightening during stress maneuvers in complete tears. ${ }^{10} \mathrm{~A}$ chronic injury may appear as ligamentous thickening, attenuation, or nonvisualization. Avulsion injuries may show tiny echogenic shadowing fragments of bone.

\section{Lateral Collateral Ligament Complex}

The lateral collateral ligament complex of the ankle consists of the anterior talofibular ligament (ATFL), the posterior talofibular ligament (PTFL), and the calcaneofibular ligament (CFL; Figure 1). For evaluation of the lateral ligaments, the patient is asked to internally rotate the hip or turn up slightly onto the contralateral side to expose the lateral ankle.

The ATFL extends from the anterolateral border of the lateral malleolus to the lateral surface of the talar neck and restrains anterior talar motion. ${ }^{8}$ To visualize the ATFL, the ankle is slightly inverted, and the transducer is placed parallel to the sole of the foot between the distal tip of the lateral malleolus and the talus. The ligament will often appear hypoechoic initially because of anisotropy, so the transducer must then be angled perpendicular to the ligament fibers (Figure 2). ${ }^{9}$ In addition, the ATFL most commonly consists of two bands or fascicles, which should not be interpreted as a ligament tear. ${ }^{11}$ The ATFL may also be imaged with US dynamically, analogous to the anterior drawer sign, to visualize discontinuity of the ATFL ligament fibers. ${ }^{12}$

The PTFL extends horizontally from the lateral tubercle of the posterior process of the talus to the posterior aspect of the lateral malleolus (the fibular malleolar fossa; Figure 3). ${ }^{8}$ The PTFL is the strongest and deepest ligament of the lateral collateral ligament complex and is rarely torn in ankle sprains. Given that it is uncommonly injured, its deep location, and poor acoustic window, the PTFL is uncommonly assessed by US, although it can be seen. ${ }^{9}$

The CFL extends from the tip of the lateral malleolus to the trochlear eminence on the lateral surface of the calcaneus. ${ }^{8}$ Its role is to stabilize the subtalar joint. With the ankle positioned on its medial aspect and with a dorsiflexed foot (which tightens the CFL), the transducer is placed in an oblique plane between the fibular tip and the posterior aspect of the heel. Here, the CFL can be identified deep to the peroneal tendons (Figure 4). Lack of elevation of the peroneal tendons with dorsiflexion is an indirect indicator of a CFL tear. ${ }^{13}$ The normal CFL may appear hypoechoic because of anisotropy when the foot is in a neutral position, which is a potential pitfall.

The ATFL is the most commonly injured ligament in the body (Figures 5 and 6), isolated or in

Figure 4. Ultrasonographic appearance of the CFL. With the ankle on its medial aspect with the foot dorsiflexed, place the transducer in an oblique plane between the fibular tip and the posterior aspect of the heel (A). The CFL (arrowheads in B) can be identified deep to the peroneal tendons (asterisk). The normal CFL may appear hypoechoic (arrowheads) because of anisotropy when the foot is in a neutral position (C). Axial T2-weighted MR image shows a normal CFL (arrow). CAL indicates calcaneus.
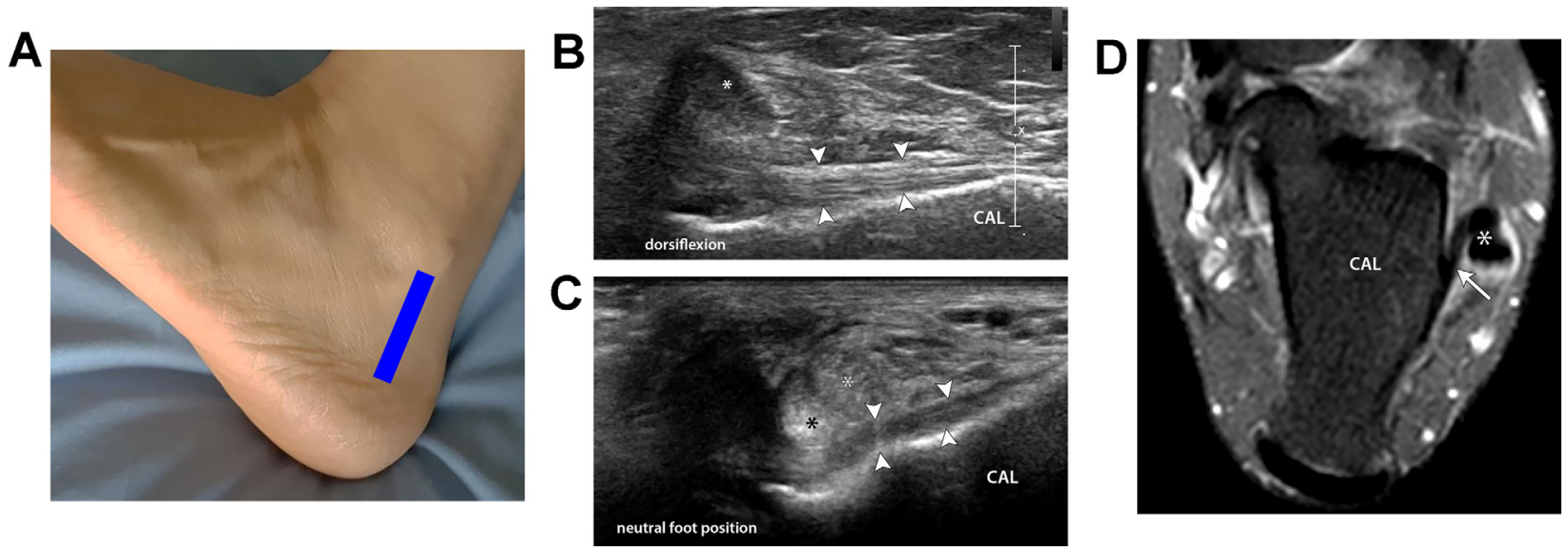
Figure 5. Images from a 29-year-old man with remote partial ATFL injury. Sonogram longitudinal to the ATFL (A) shows hypoechoic thickening without discontinuity (arrowheads). Axial T2-weighted (B) and T1 weighted (C) MR images from the same patient show similar findings (arrowheads) with an increased internal signal and adjacent edema. LM indicates lateral malleolus.

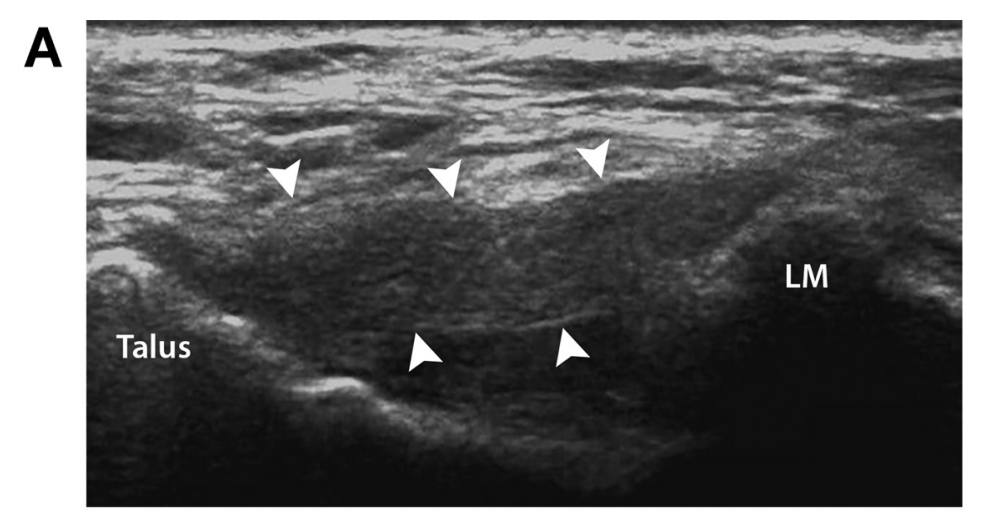

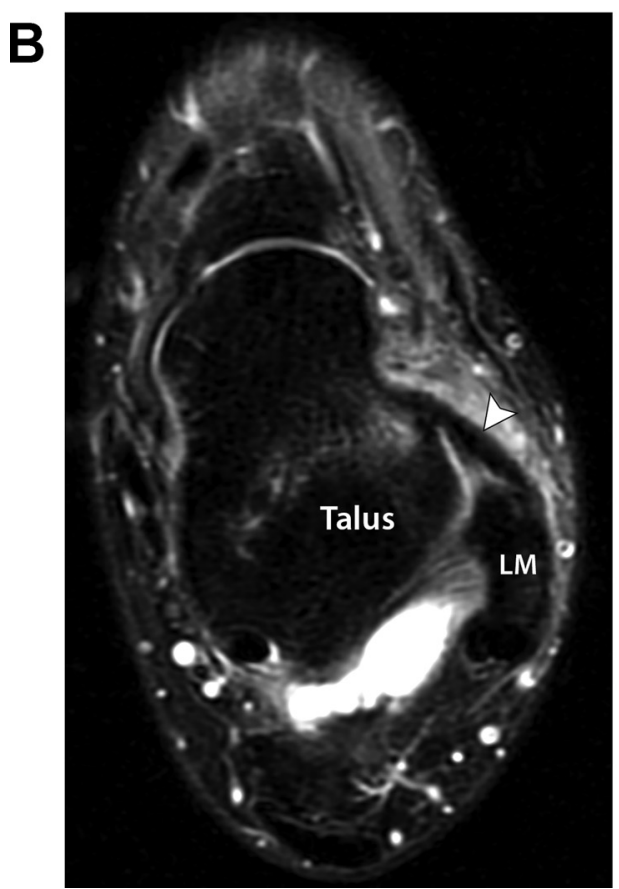

combination with a CFL tear in up to $70 \%$ of cases (Figures 7 and 8). ${ }^{2}$ The CFL is usually sequentially torn after the ATFL is torn during inversion injuries, so an isolated tear of the CFL is unlikely when a normal ATFL is identified. Complete CFL tears may be accompanied by fluid in the overlying peroneal tendon sheath. ${ }^{1}$ The accuracy of US in the diagnosis of ATFL and CFL tears has been reported to range from $87 \%$ to $100 \%{ }^{8}$ Ultrasonography was shown to have an accuracy rate of $91 \%$ in the diagnosis of ATFL

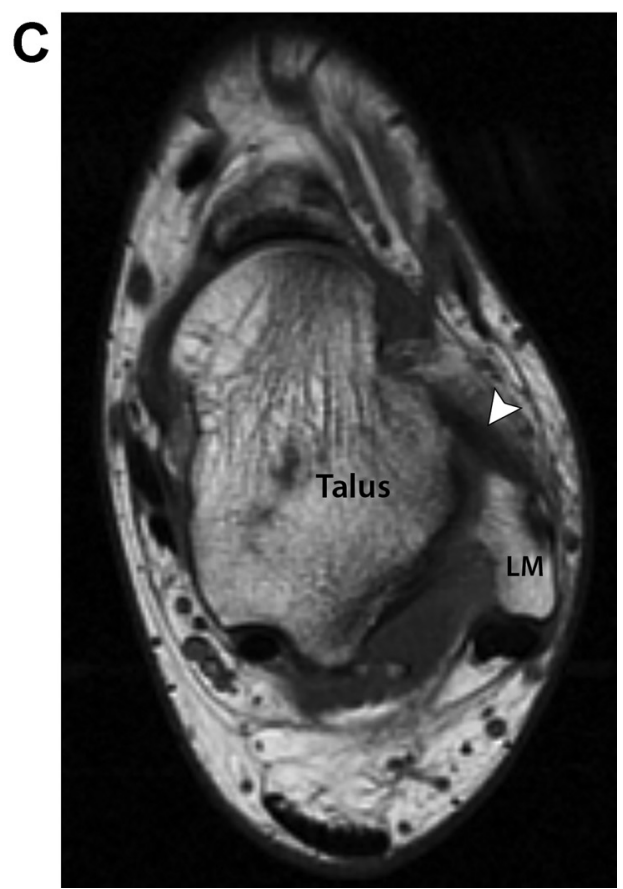

injuries versus $97 \%$ for MRI. ${ }^{14}$ It was also shown to be highly sensitive and specific for chronic lateral ankle ligament injuries. ${ }^{15}$

\section{Medial Collateral Ligament (Deltoid) Complex}

The medial collateral ligament or deltoid complex is the primary medial stabilizer of the ankle and 
Figure 6. Complete ATFL tears. Sonogram longitudinal to the ATFL from a 38-year-old man (A) shows absence of the ATFL with a fluid-filled gap (arrowhead). Axial T2-weight MR image from a different patient, a 21-year-old woman (B), shows similar findings of a complete ATFL tear. LM indicates lateral malleolus.
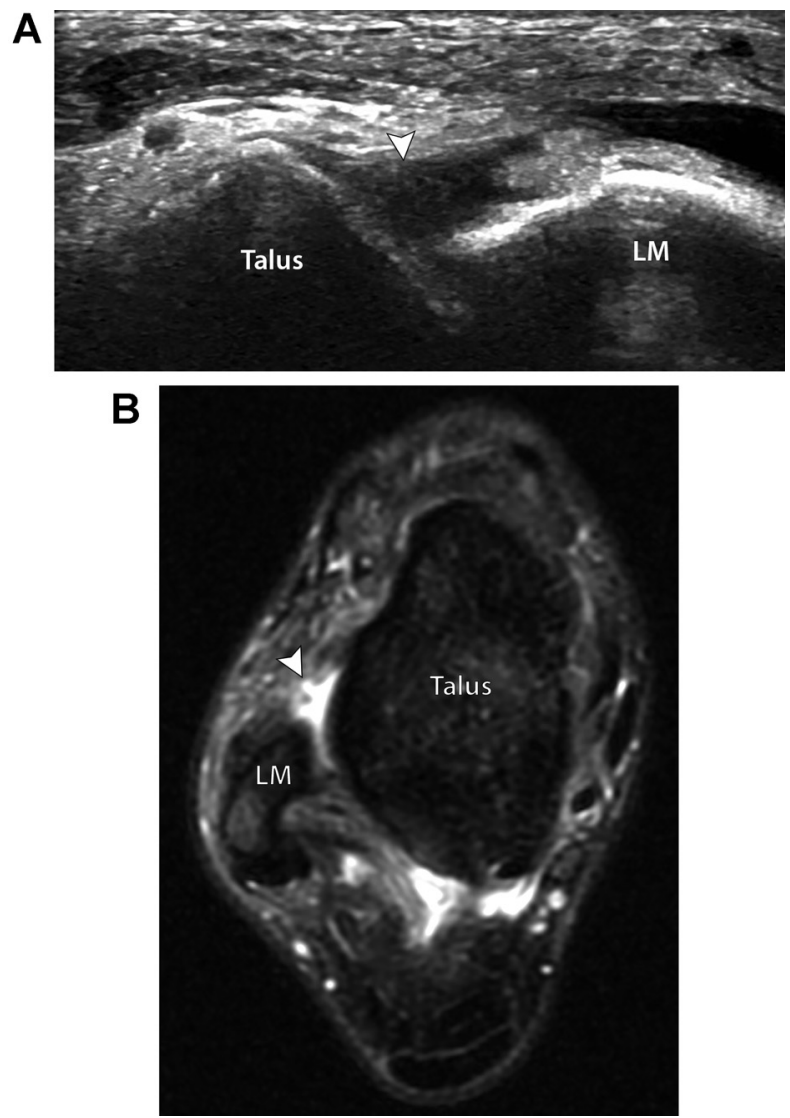

consists of two distinct layers (Figure 9). The deep layer has talar attachments extending from the medial malleolus and consists of the anterior and posterior tibiotalar ligaments. ${ }^{8,16}$ The superficial layer has variable attachments, extending from the medial malleolus to the navicular bone (tibionavicular ligament), the superomedial spring ligament (tibiospring ligament), and the calcaneus (tibiocalcaneal ligament). ${ }^{8}$ The posterior tibiotalar ligament has also been described has having both superficial and deep components. ${ }^{17}$ For evaluation of the medial ligaments, the patient is asked to externally rotate the hip or turn up onto the ipsilateral side to expose the medial ankle. The transducer is initially placed in the coronal plane at the level of the medial
Figure 7. Partial CFL tears. Sonogram from a 28-year-old man (A) shows hypoechoic thickening of the CFL (arrowheads) deep to the peroneal tendons (asterisks). Corresponding axial T2-weighted MR image (B) shows similar findings with surrounding edema in a different patient. CAL indicates calcaneus.
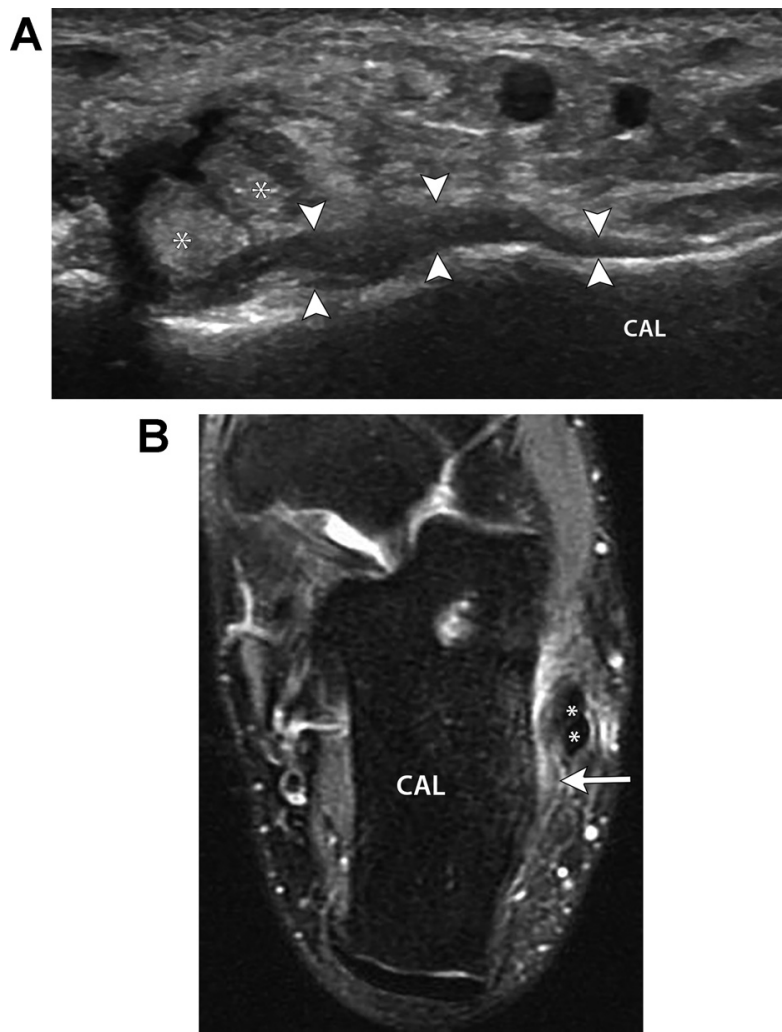

malleolus and the inferior aspect rotated slightly posterior. The tibiocalcaneal component of the deltoid ligament can be identified extending from the tibia to the calcaneus (Figure 10). To visualize the posterior tibiotalar component, the foot is dorsiflexed, and the distal aspect of the transducer is rotated posteriorly while the proximal aspect is fixed to the medial malleolus (Figure 10). Of note, the tibiotalar ligament complex may appear hypoechoic because of its angulation and interspersed fat; this appearance should not be mistaken for a tear. ${ }^{8}$ Within the medial collateral ligament complex, the posterior tibiotalar ligament is considered the strongest component. ${ }^{16,18}$ Next, the proximal aspect of the transducer is fixed to the medial malleolus, and the distal aspect is rotated into the coronal plane to identify the more superficial tibiospring, and then rotated 
Figure 8. Complete CFL tear. Sonogram (A) shows hypoechoic thickening of the CFL (arrows) with a defect of the distal CFL (arrowheads). Corresponding axial T2-weighted MR image from a 22-year-old woman (B) reveals a similar finding with an additional tear of the peroneus brevis (arrow). Asterisks indicate peroneal longus; and CAL, calcaneus.
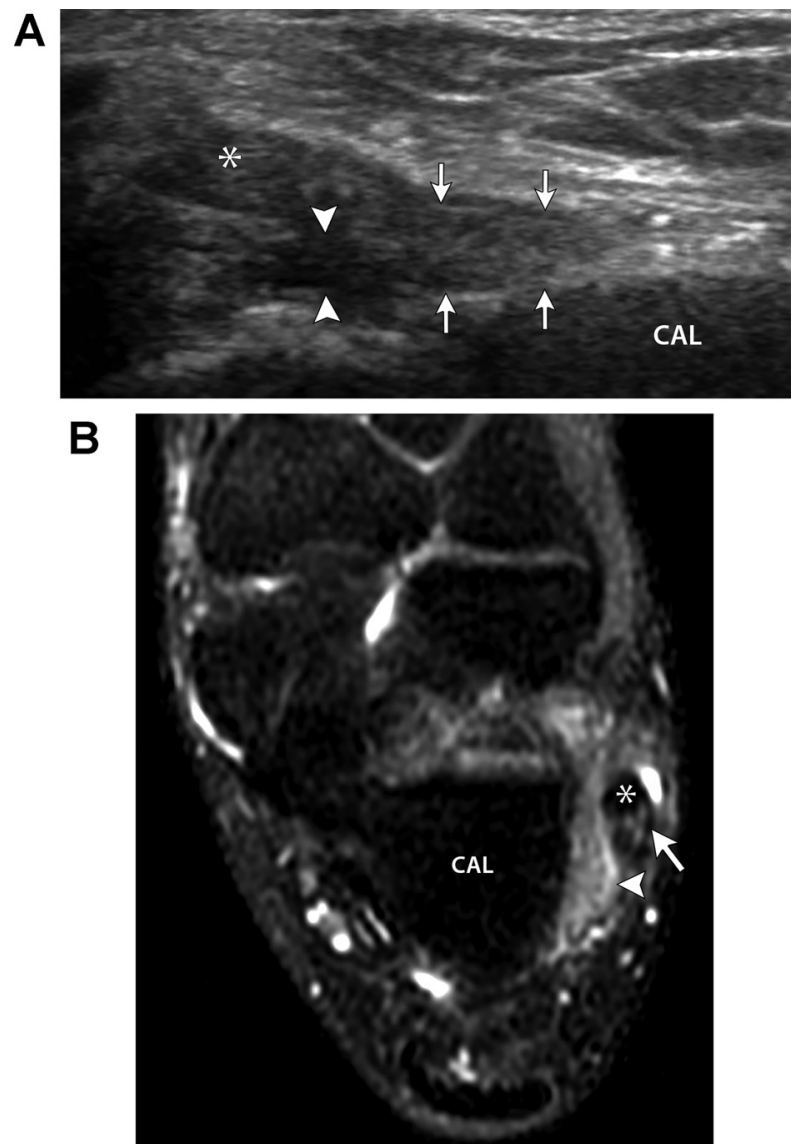

more anteriorly to identify the tibionavicular and deeper anterior tibiotalar components (Figure 11).

The deltoid ligament complex is less frequently injured than the lateral complex but may be injured during eversion stress. A US evaluation of deltoid ligament tears can be challenging given its complexity and variability. ${ }^{19}$ Deltoid ligament injuries typically produce hypoechoic swelling that involves several components with or without frank disruption and avulsion fracture fragments (Figure 12). Stripping of the medial malleolus fascial sleeve, which is composed of the tibial attachments of the superficial deltoid ligament and flexor retinaculum, and the periosteum has been described as an important sign
Figure 9. Illustrations of the normal anatomy of the medial (deltoid) ligament complex. Medial view (A) shows the tibionavicular ligament (light blue), tibiospring ligament (yellow), tibiocalcaneal ligament (green), anterior tibiotalar ligament (orange), posterior tibiotalar ligament (purple), and superomedial calcaneonavicular ligament (blue with asterisk). Coronal view (B) shows the superficial (arrow) and deep (arrowhead) layers of the medial complex. CAL indicates calcaneus, and N, navicular.

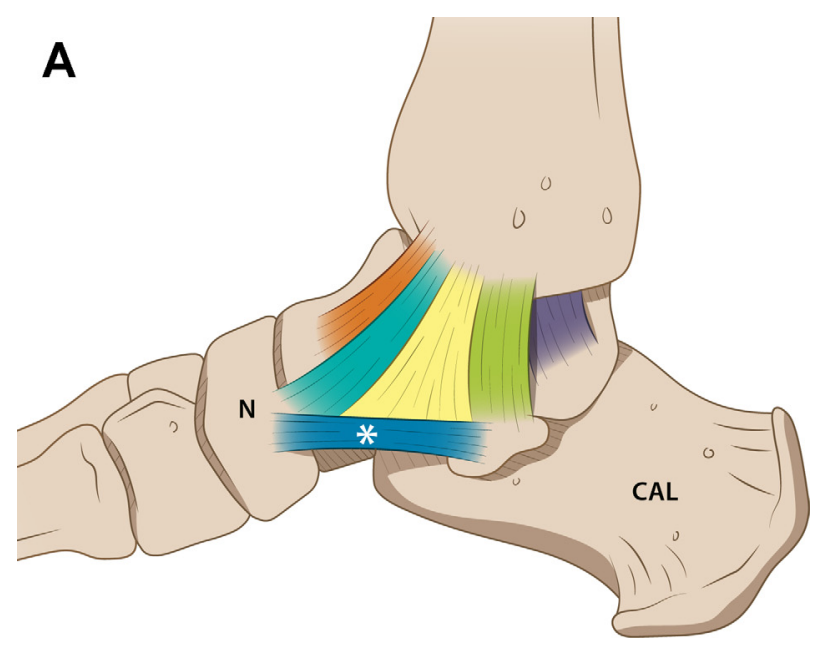

B

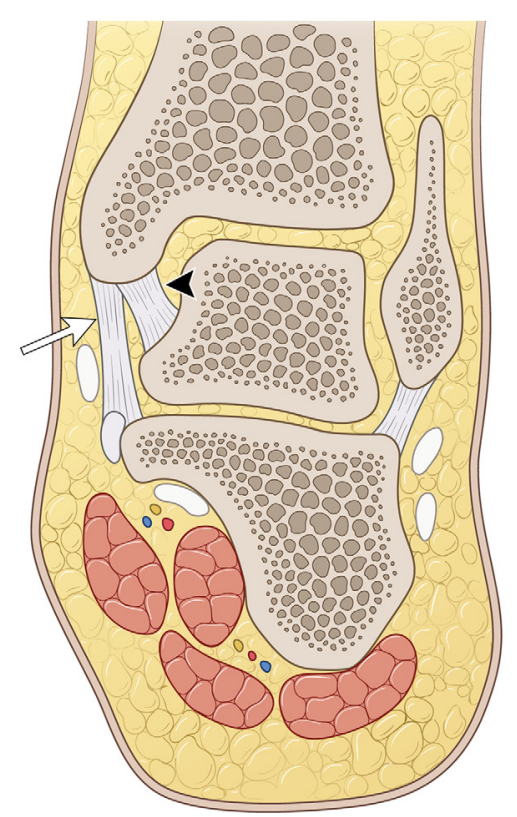

that indicates a superficial deltoid ligament tear. ${ }^{20}$ Deep ligament tears are more common than superficial ligament tears, and partial tears are more common than complete tears (Figures 12 and 13). ${ }^{18,21,22}$ When the deltoid ligament is acutely 
Figure 10. Ultrasonographic anatomy of the medial (deltoid) complex. By placing the superior edge of the transducer over the medial malleolus and rotating the distal aspect of the transducer posterior or parallel (A), different components of the medial (deltoid) complex can be identified: posterior tibiotalar ligament (arrowheads in B) and tibiocalcaneal ligament (arrowheads in C). Coronal T2-weight MR image (D) shows an intact posterior tibiotalar ligament (arrowheads), with the large arrow pointing to the posterior tibialis tendon. CAL indicates calcaneus; and MM, medial malleolus.
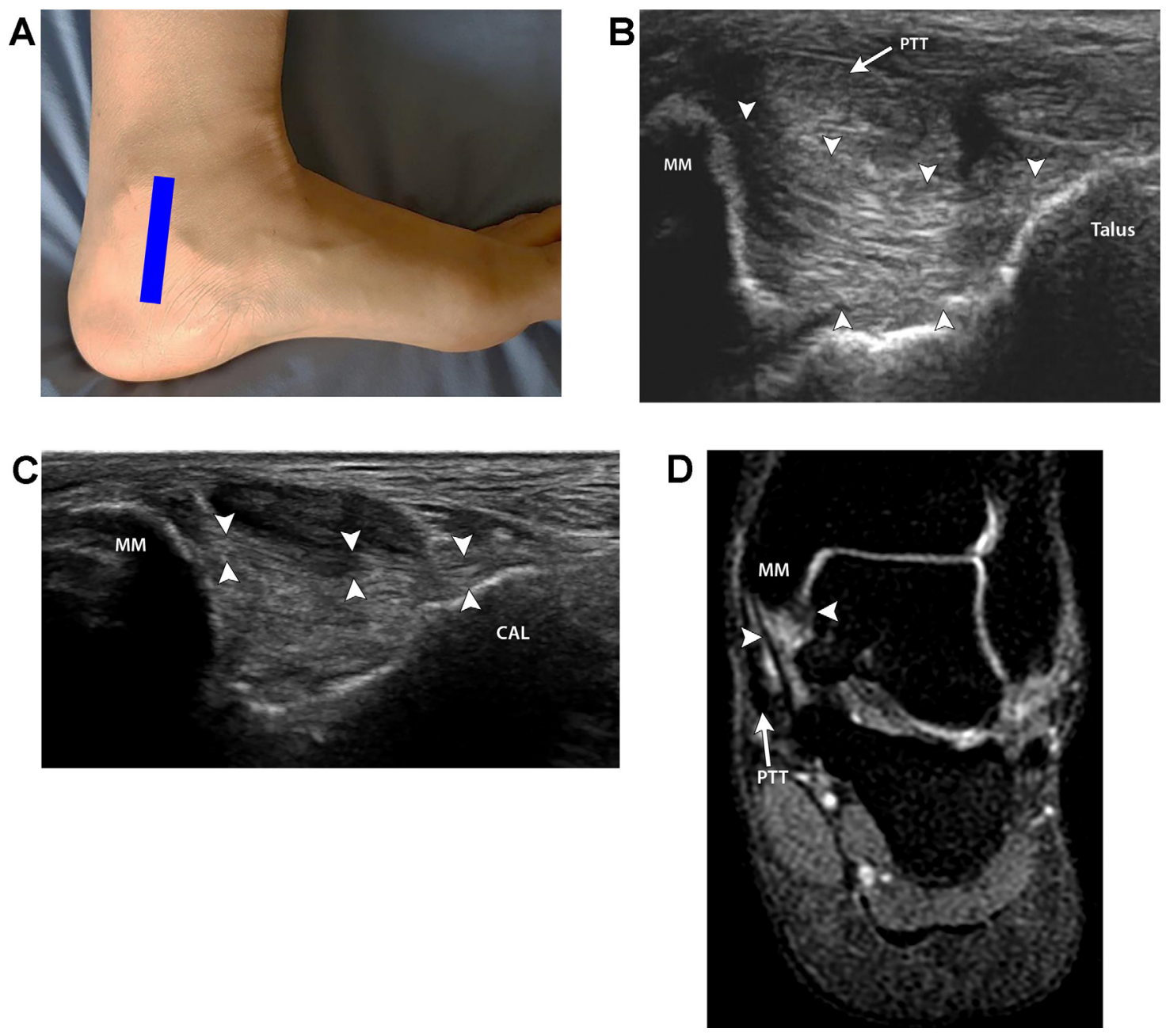

torn, the possibility of associated lateral malleolar fractures or distal tibiofibular ligament tears should be evaluated. ${ }^{1,7}$ In the setting of Weber type B and C fractures, US was found to have $100 \%$ sensitivity for the detection of complete rupture of the deltoid ligament but only sensitivity of $50 \%$ for partial ruptures; thus, it may be helpful but not entirely replace MRI for the assessment of medial instability in this clinical setting. ${ }^{23}$ Another study demonstrated $100 \%$ sensitivity and specificity of US for medial deltoid rupture with a $100 \%$ correlation between US and arthroscopy in supination external rotation fractures of the ankle, thus possibly obviating the need for stress radiographs. ${ }^{24}$

\section{Spring Ligament Complex (Calcaneonavicular Ligament)}

The spring ligament complex is composed of the superomedial, medioplantar, and inferoplantar calcaneonavicular ligaments (Figure 14). ${ }^{16,25}$ The spring 
Figure 11. Normal US and MRI appearance of the medial (deltoid) complex, continued. The transducer is rotated into the coronal plane, showing the tibiospring ligament (arrowheads in $\mathbf{A}$ ), and then rotated anteriorly to show the anterior tibionavicular ligament (arrowheads in B) and anterior tibiotalar ligament (arrows in B). Coronal T2-weighted fat-saturated MR image (C) shows the normal tibiospring ligament (arrow) and partial visualization of the anterior tibiotalar ligament (white arrowhead). Axial T2-weighted fat-saturated MR image (D) shows the normal superficial (arrowhead) and deep (arrow) fibers of the medial (deltoid) complex. Asterisks indicate spring ligament in $\mathbf{A}$ and $\mathbf{C}$; MM, medial malleolus; N, navicular; and PTT, posterior tibialis tendon (black arrowhead in $\mathbf{C}$ ).
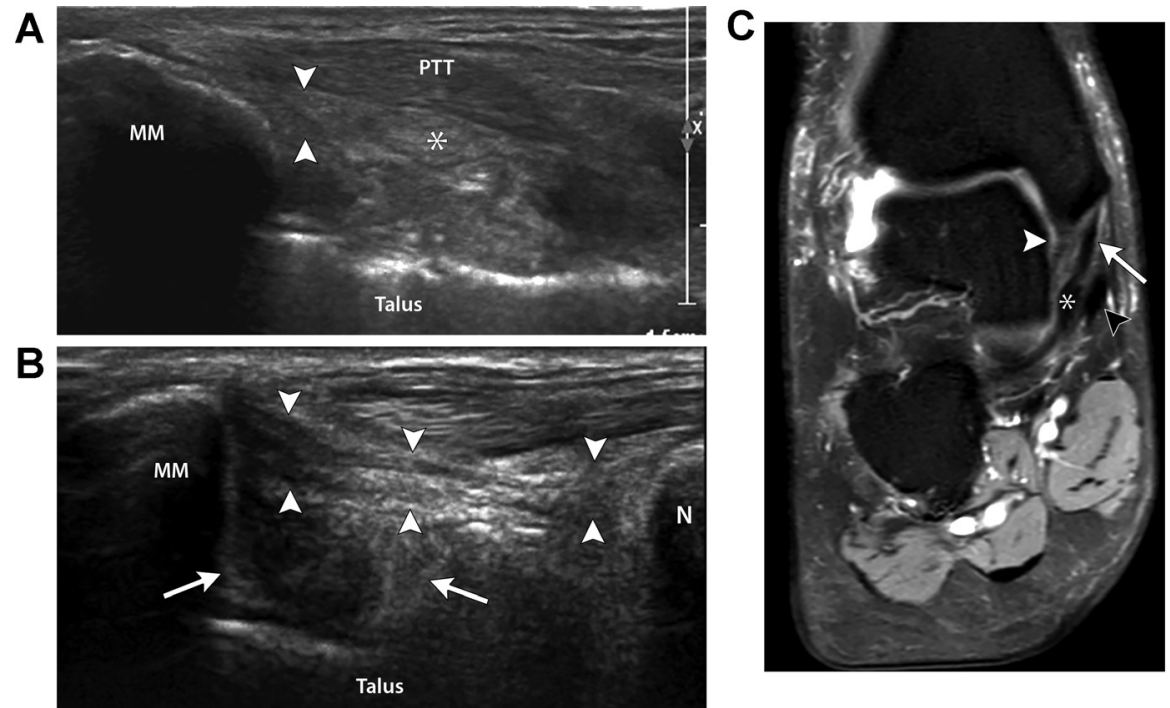

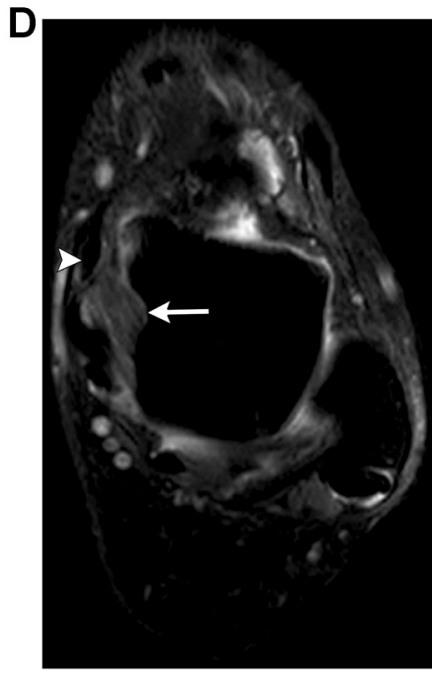

ligament is one of the major stabilizers of the longitudinal arch and for supporting the head of the talus. $^{8,26}$

With the foot rotated externally with dorsal extension, the transducer is initially placed in the transverse plane inferior to the medial malleolus and over the sustentaculum tali. The transducer is then moved anteriorly and angled superiorly toward the talar head, allowing the superomedial calcaneonavicular ligament to be identified in long axis (Figure 15). ${ }^{27}$ The medioplantar and inferoplantar calcaneonavicular ligaments are not consistently visualized on US because of their deep and complex course. ${ }^{8}$ The superomedial calcaneonavicular ligament thickness has been described as 1.9 to $4.7 \mathrm{~mm}^{25}$

The superomedial calcaneonavicular ligament is the most likely to be injured (Figure 16). Disorders of the calcaneonavicular ligament are associated with tibialis posterior tendon tears, both of which contribute to flatfoot deformity. ${ }^{28}$ Injury to the spring ligament appears as thickening, thinning, or frank discontinuity. ${ }^{25,28}$ Ultrasonography is an effective tool for assessing spring ligament abnormalities in patients with symptomatic posterior tibialis tendon dysfunction. $^{28}$

\section{Ankle Syndesmosis}

The ankle syndesmosis is stabilized by the anterior inferior tibiofibular ligament (AITFL), the posterior inferior tibiofibular ligament (PITFL), the inferior transverse ligament, and the distal interosseous ligament (Figure 17). ${ }^{16}$ The interosseous membrane is found more proximally between the tibial and fibular diaphysis, and the distal aspect of the interosseous membrane is contiguous with the interosseous ligament. $^{29}$

With the ankle slightly inverted, the transducer is placed with the posterior edge on the lateral malleolus, and its anterior edge is rotated upward along the distal tibia. Here, the AITFL may be identified, extending obliquely downward and laterally from the anterior tibial tubercle to the anterior border of the distal fibular shaft and lateral malleolus (Figure 18). ${ }^{8}$ The normal thickness of the AITFL ranges from 2.6 
Figure 12. Medial (deltoid) complex injury. Sonogram (A) from a patient after acute injury shows heterogeneous hypoechoic discontinuity of the deltoid ligament with an adjacent fracture fragment (black arrow), involving both the superficial (arrowhead) and deep (white arrow) components. Axial T2-weighted MR images (B and C) from a different patient, a 21-year-old man, show a tear involving both superficial (arrowheads) and deep (white arrows) components, with a fracture fragment (small white arrow in $\mathbf{C}$ ) and intact posterior tibiotalar ligament (asterisks in $\mathbf{B}$ and $\mathbf{C}$ ).

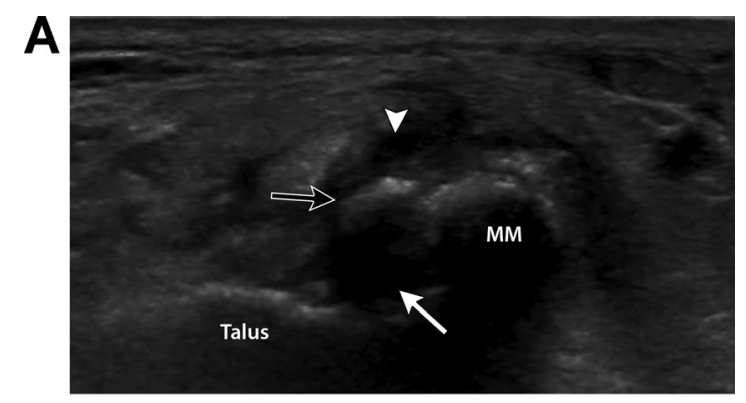

B

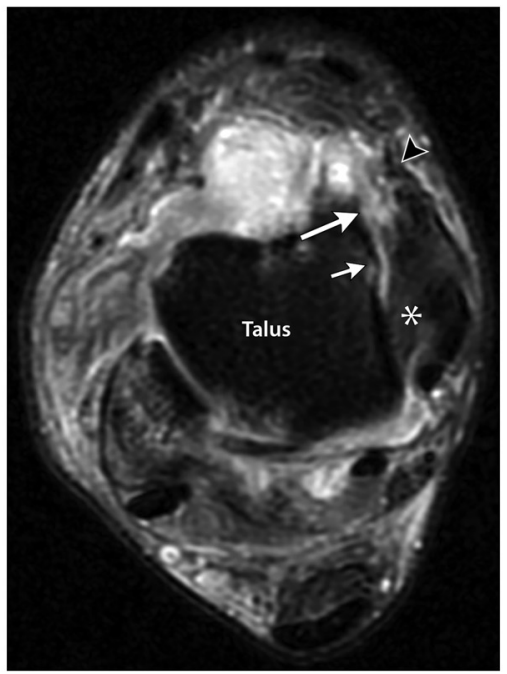

C

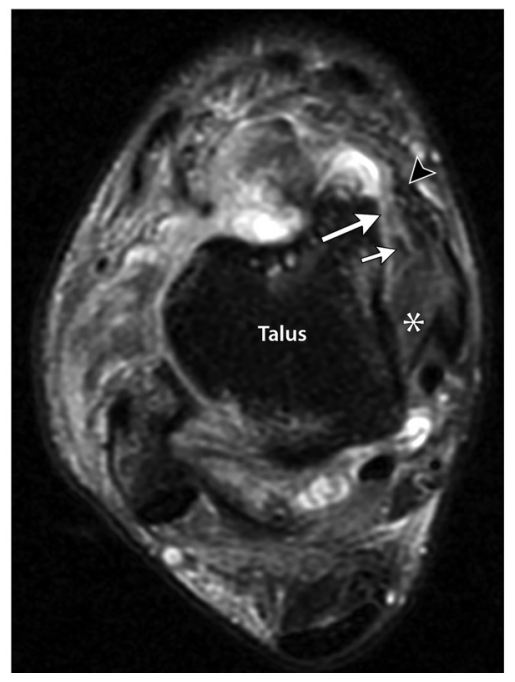

Figure 13. Medial (deltoid) complex injury, continued. Sonogram (A) after acute injury shows heterogeneous hypoechoic discontinuity of the deltoid ligament (white arrow) with an adjacent fracture fragment (black arrow), involving the deep component, predominantly the anterior tibiotalar ligament. The superficial complex (white arrowhead) remains intact. Coronal (B) and axial (C) T2-weighted MR images from a different patient, a 36-year-old man, show a tear involving the deep component (white arrows), predominantly the posterior tibiotalar ligament with an adjacent fracture fragment (black arrow).

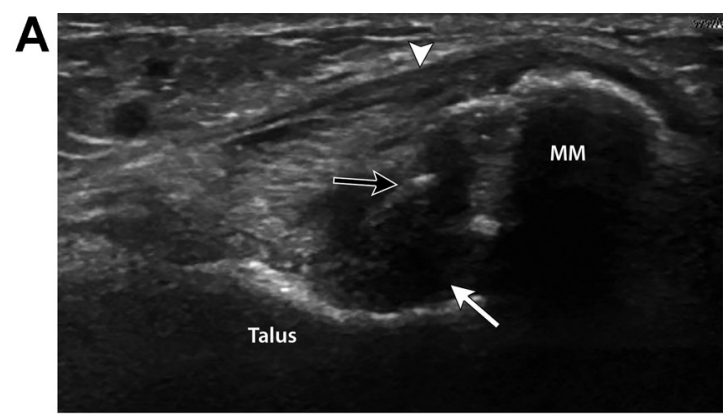

B
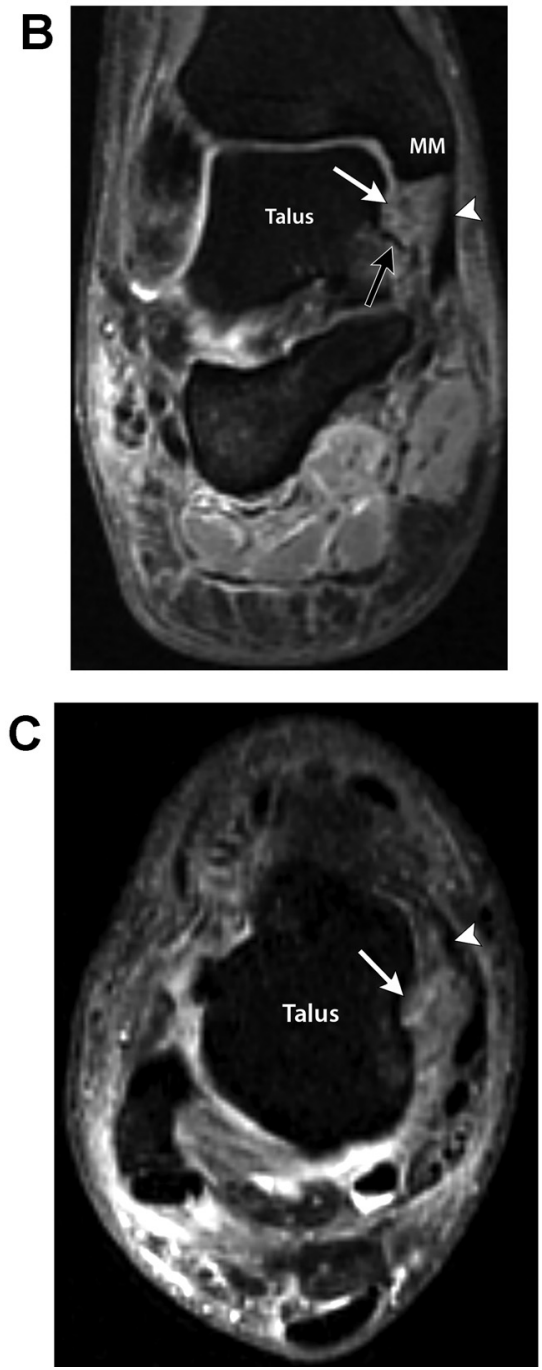
Figure 14. Illustrations of the normal anatomy of the spring ligament complex (calcaneonavicular ligament): superomedial calcaneonavicular ligament (asterisk in $\mathbf{A}$ ), medioplantar oblique calcaneonavicular ligament (arrows in $\mathbf{A}$ and $\mathbf{B}$ ), and inferoplantar longitudinal calcaneonavicular ligament (arrowheads in $\mathbf{A}$ and $\mathbf{B}$ ).
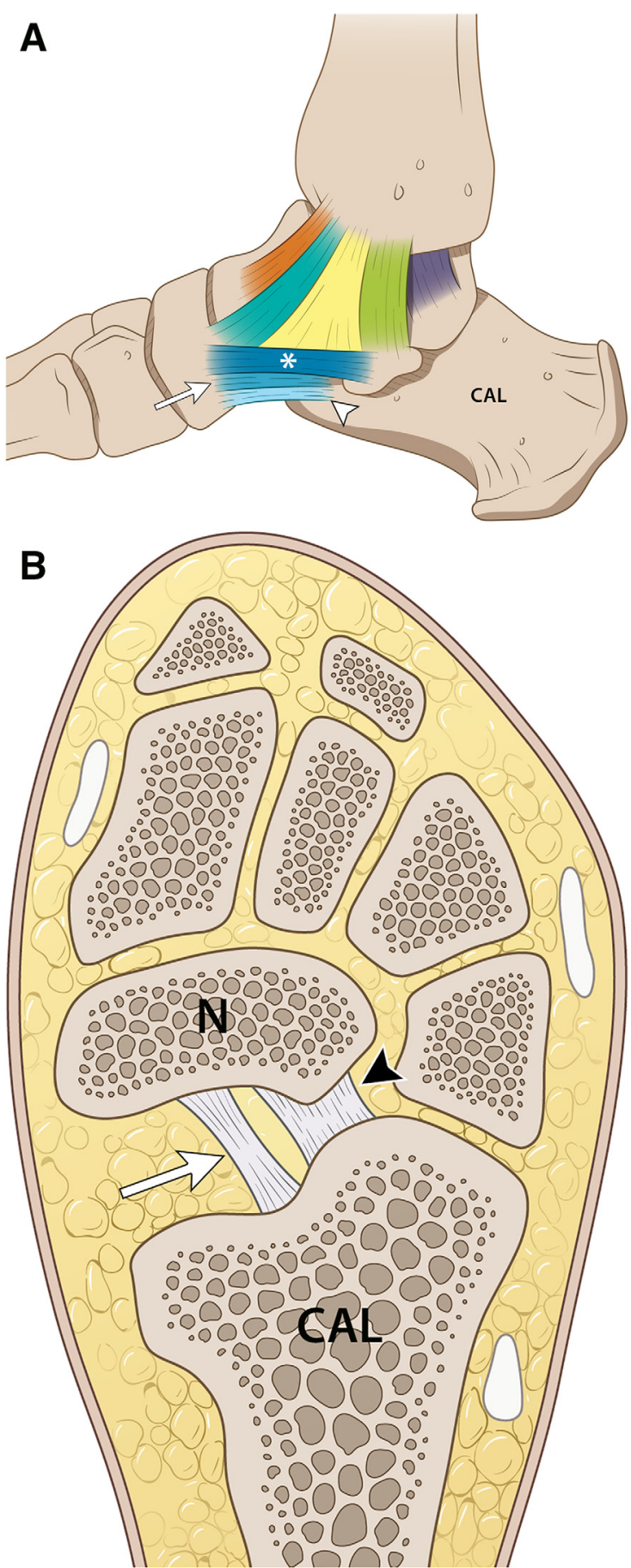

Figure 15. Normal US appearance of the spring ligament. By rotating the foot externally with dorsal extension, the superomedial calcaneonavicular ligament is imaged by placing the transducer parallel to the plantar surface inferior to the medial malleolus over the sustentaculum tali, with the other end of the transducer slightly tilted superiorly over the talar head toward the medial aspect of the navicular bone (A). Sonogram shows identification of the ligament as a hyperechoic fibrillar structure (arrowheads in $\mathbf{B}$ ) deep to the posterior tibialis tendon (asterisks in $\mathbf{B}$ and $\mathbf{C}$ ). Corresponding axial T2-weighted MR image (C) shows a normal superomedial calcaneonavicular ligament (black arrowheads). $\mathrm{N}$ indicates navicular.

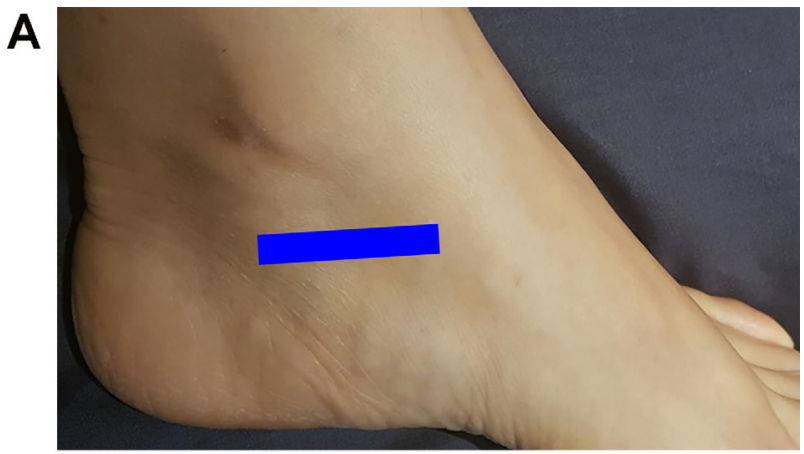

B

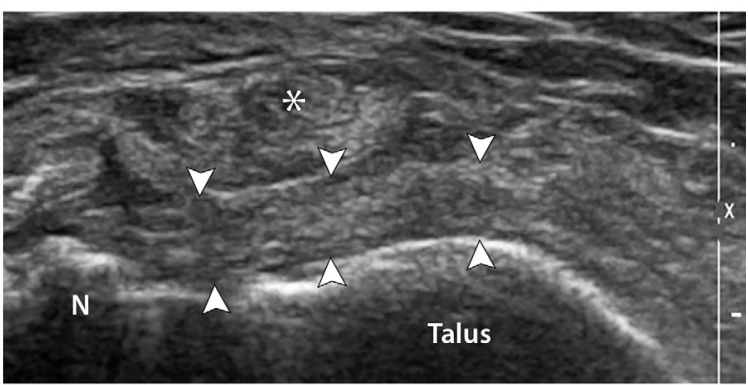

C

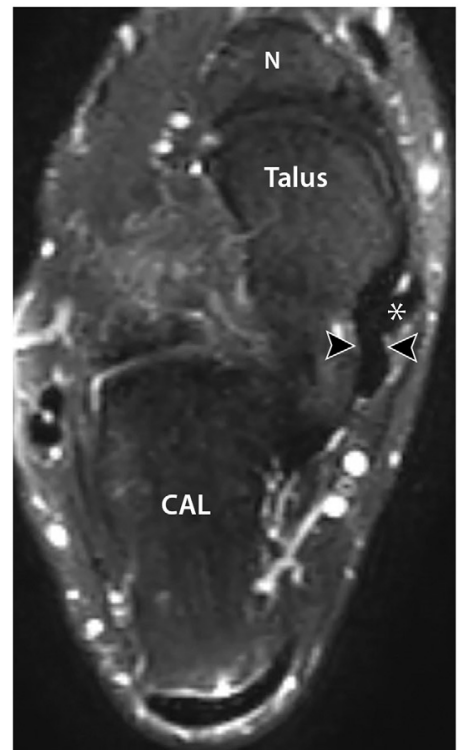


Figure 16. Images from a 29-year-old man with superomedial calcaneonavicular ligament injury. Sonogram (A) longitudinal to the superomedial calcaneonavicular ligament in a patient with acute injury shows abnormal hypoechoic thickening in the distal superomedial calcaneonavicular ligament (arrowheads). Corresponding axial T2-weighted MR image (B) from the same patient shows similar findings with an abnormal signal (arrowhead). Asterisks indicate posterior tibialis tendon; and N, navicular.
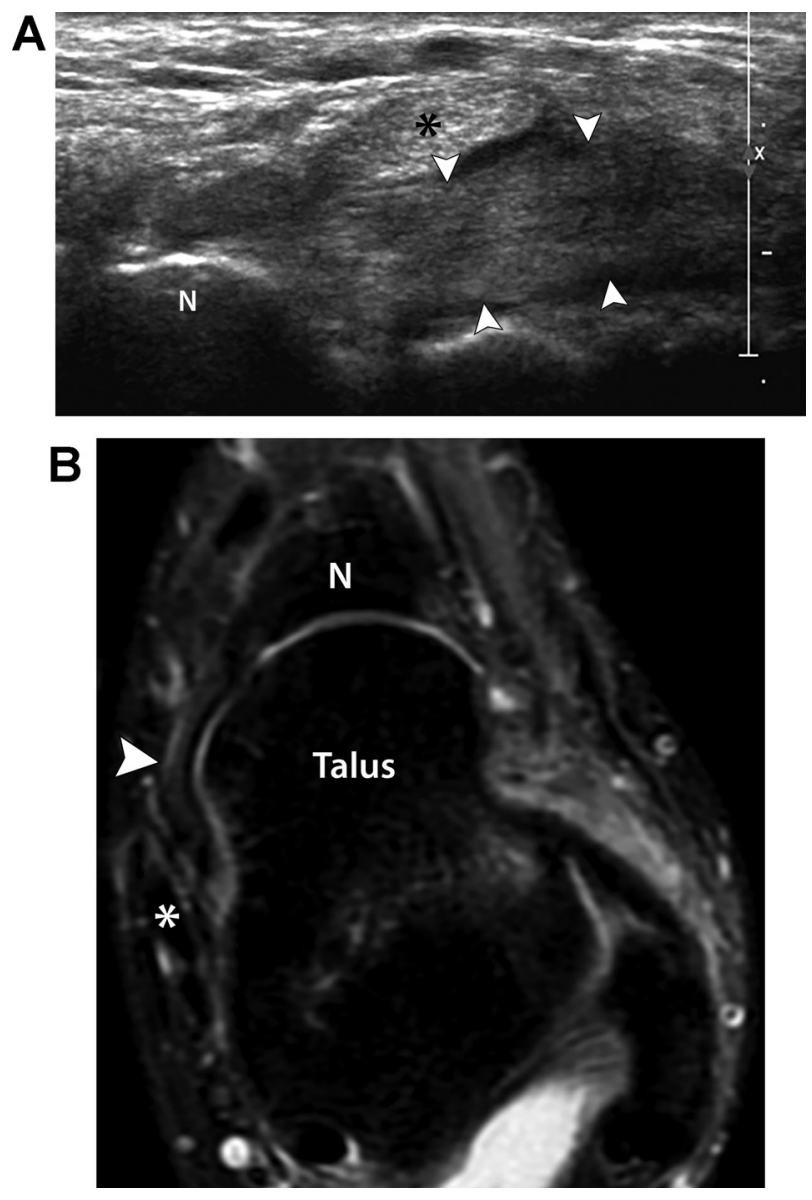

to $4 \mathrm{~mm}$. The AITFL consists of multiple fascicles

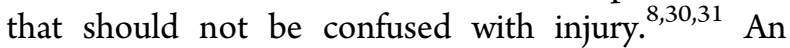
accessory AITFL (or Bassett ligament) is visualized in up to $94 \%$ of patients and may be abnormally thickened when associated with anterolateral ankle impingement. $^{32}$ The distal interosseous membrane may be imaged by placing the transducer in an axial plane between the distal tibia and fibula above the joint from an anterior approach (Figure 19), with the interosseous ligament identified at its most inferior extent proximal to the tibial plafond. The posterior
Figure 17. Illustration of the normal ankle syndesmosis: AITFL (arrows), PITFL (arrowhead), and distal interosseous membrane and ligament (asterisk).
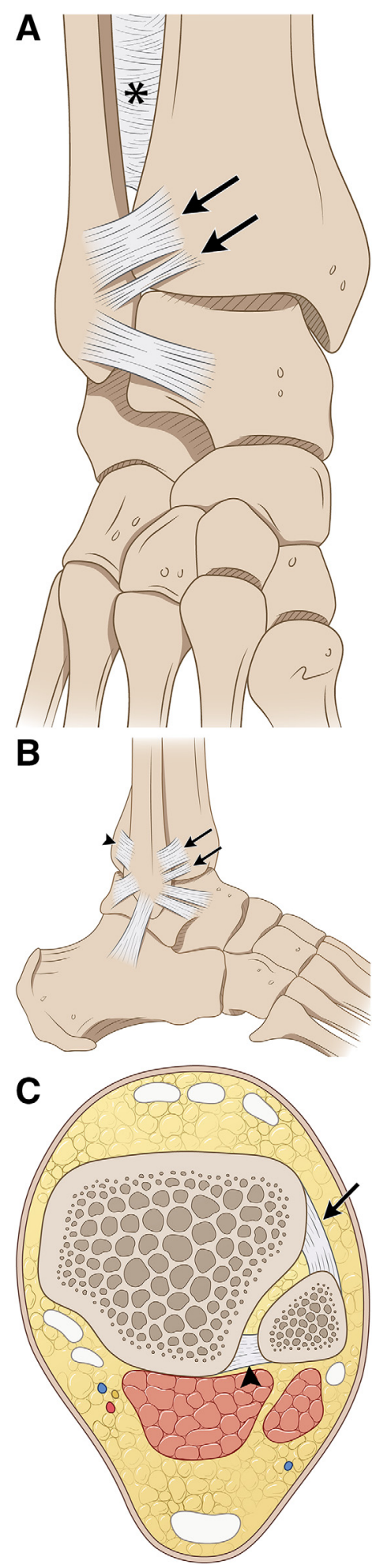
Figure 18. Ultrasonographic and MRI appearance of the normal AITFL. With the ankle slightly inverted, the transducer is placed with the posterior edge on the lateral malleolus, and its anterior edge is rotated upward (A). The AITFL can be seen as a hyperechoic fibrillar band (arrowheads in B). Lateral malleolus (LM). Corresponding axial T2-weighted MR image shows normal AITFL (arrowhead in C) and PITFL (arrow in $\mathbf{C}$ ). Asterisk indicates peroneus longus and brevis; and LM, lateral malleolus.

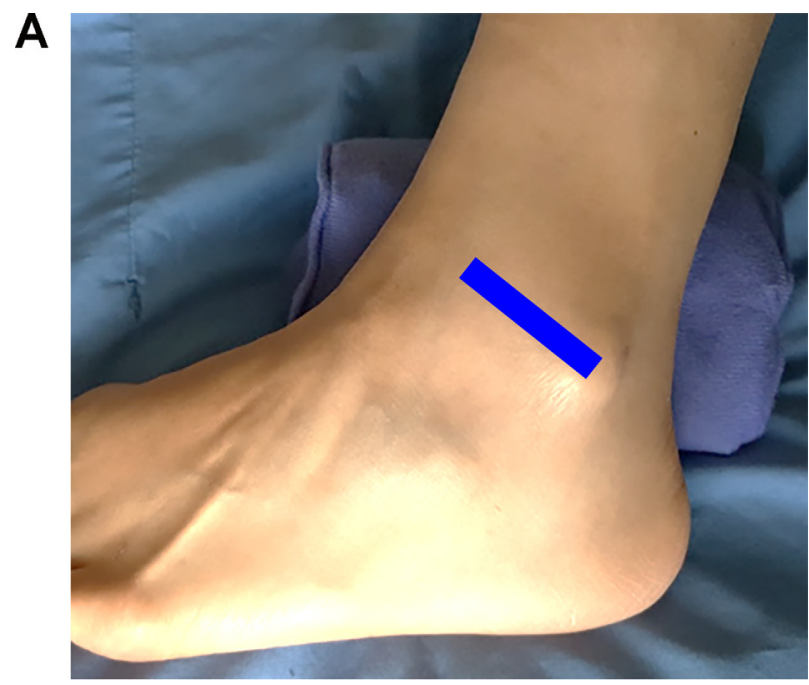

B

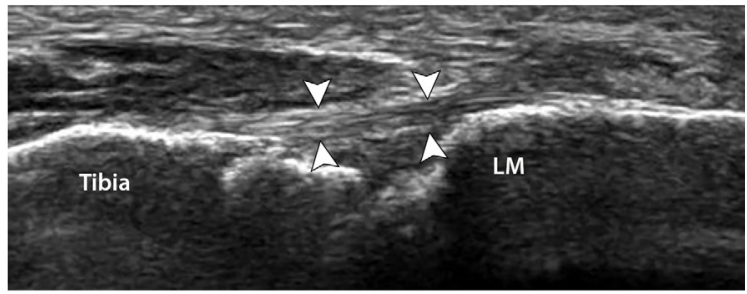

C

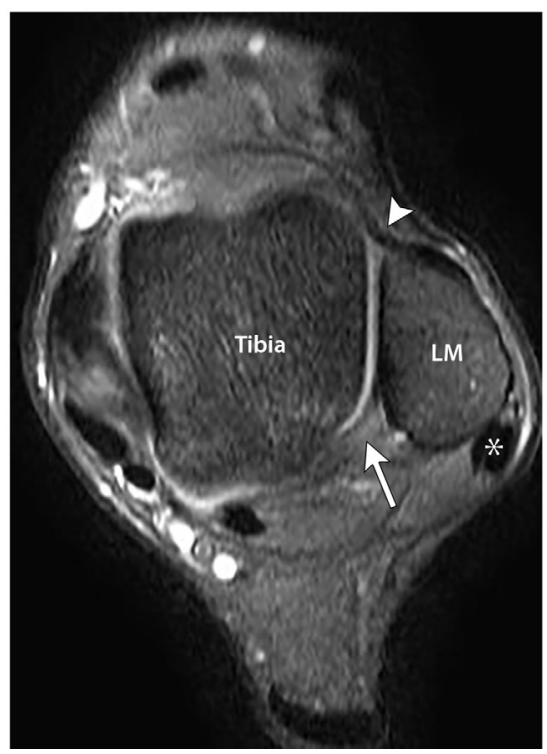

Figure 19. Normal US appearance of the distal interosseous membrane. To image the intraosseous membrane, the transducer is placed in an axial plane between the distal tibia and fibular above the joint from an anterior approach $(\mathbf{A})$. The intraosseous membrane will appear hyperechoic, thin, and taut (arrowheads in $\mathbf{B}$ ). F indicates fibula.
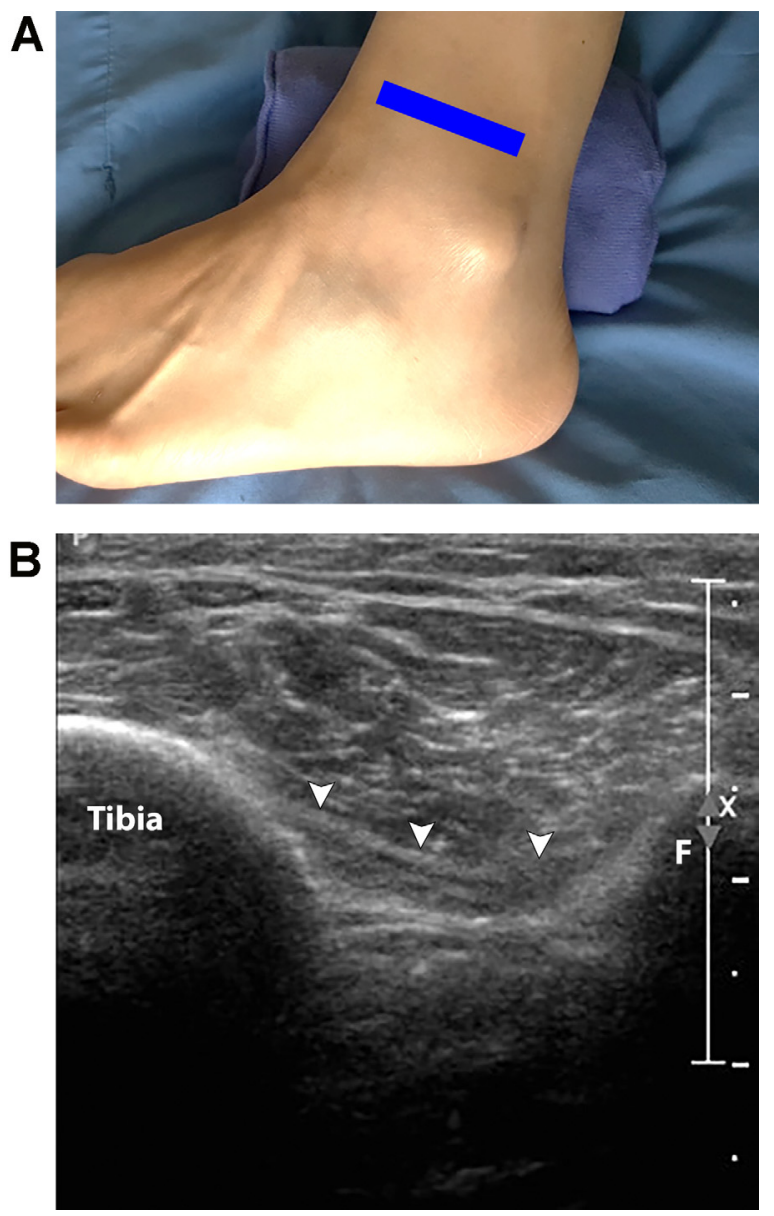

inferior tibiotalar ligament and the inferior transverse ligament are not routinely evaluated, given their depth and poor acoustic windows.

A syndesmotic ligament injury defines a high ankle sprain (Figures 20 and 21). It may be an isolated injury, occur in conjunction with other ligamentous injuries (typical lateral collateral ligament complex), or be associated with Weber B or C fractures of the distal fibula. Isolated injuries are rare but often undiagnosed. ${ }^{10}$ The accuracy of US in the evaluation of AITFL injuries is quoted at approximately $85 \%{ }^{8,33}$ A dynamic evaluation of the AITFL using 
Figure 20. Partial AITFL tear. Sonogram (A) longitudinal to the AITFL in a 26-year-old man after injury shows abnormal hypoechoic thickening (arrowheads). Corresponding axial T2-weighted MR image (B) from a different patient, a 22-year-old man, shows similar findings with an abnormal signal (arrowhead). LM indicates lateral malleolus; and MM, medial malleolus.

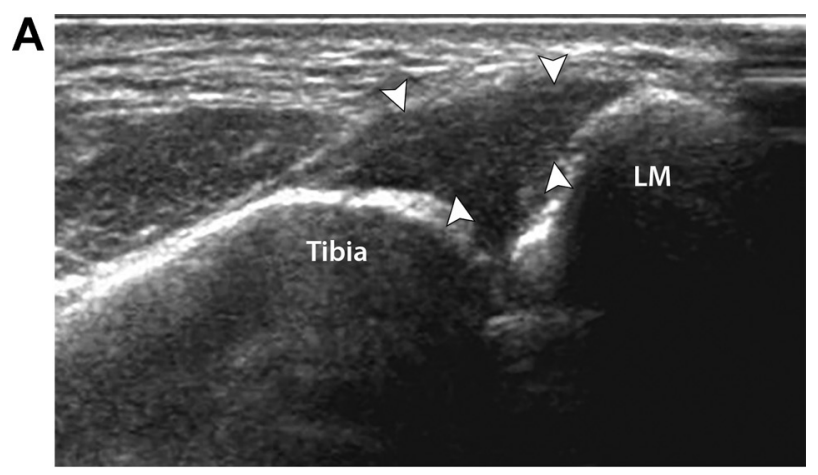

B

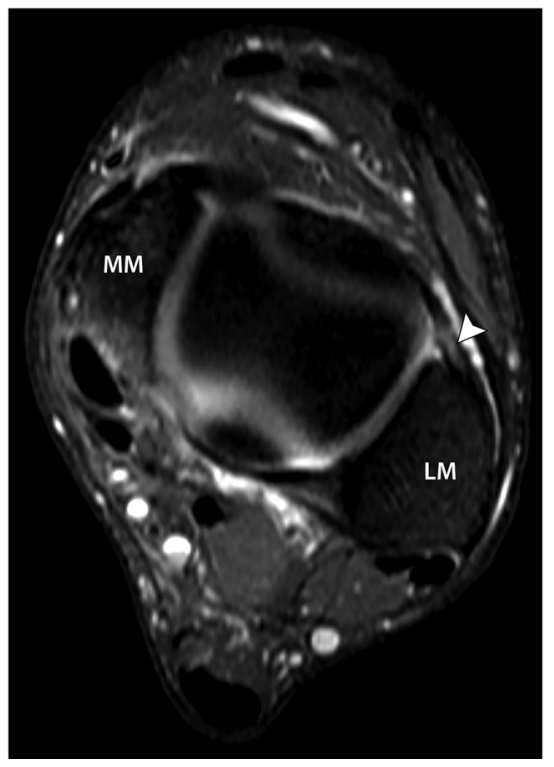

US with the ankle in eversion can show widening of the tibiofibular clear space and ligament fiber discontinuity. ${ }^{34}$

In the setting of an AITFL tear, an associated tear of the distal interosseous ligament and membrane should be considered and evaluated (Figure 22). A tear of the interosseous membrane appears as abnormal hypoechogenicity and discontinuity. ${ }^{29}$ This injury may also be associated with a proximal fibular fracture or Maisonneuve injury. ${ }^{29}$ Additionally, a joint effusion in the tibiofibular
Figure 21. Images from a 39-year-old woman with a complete AITFL tear. Sonogram (A) longitudinal to the AITFL after injury shows anechoic discontinuity (arrow) of the ligament (arrowheads). Corresponding axial T2-weighted MR image (B) from the same patient shows similar findings with an abnormal signal (arrowhead). Note that the PITFL is also torn (black arrow). LM indicates lateral malleolus.
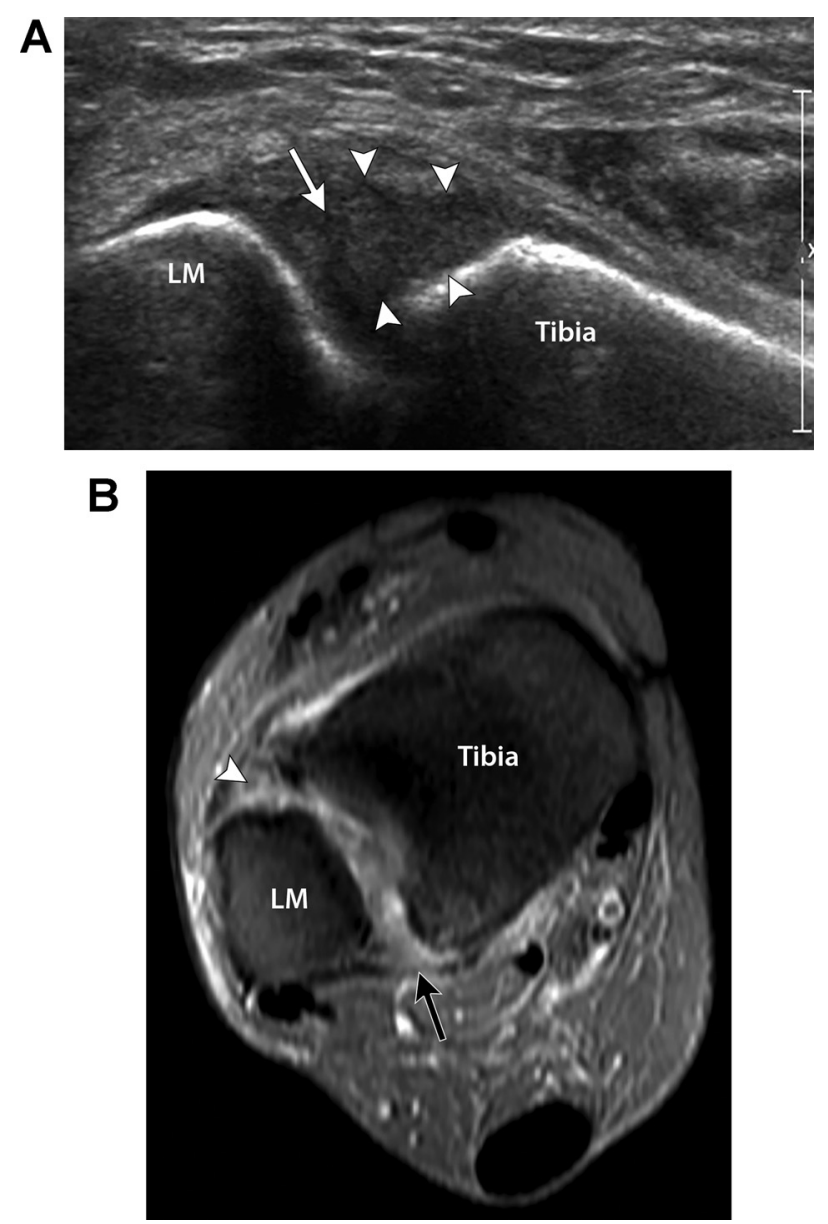

recess on MRI that extends greater than $12 \mathrm{~mm}$ proximal to the tibial plafond may suggest interosseous ligament injury. ${ }^{16}$

\section{Conclusions}

Ultrasonography provides exquisite delineation of the normal anatomy of the ankle ligaments and can be used effectively to evaluate ligament integrity. Knowledge of the normal US anatomy, 
Figure 22. Interosseous membrane injury. Transverse sonogram (A) of the anterolateral lower leg above the ankle joint from a 68-year-old man shows nonvisualization of the interosseous membrane (arrow). The contralateral leg imaged at the same location shows an intact interosseous membrane (arrowheads). Coronal T1-weighted fat-saturated image on MR arthrography (B) from a different patient, a 19-year-old man, with ankle pain shows intraarticular contrast extending greater than $12 \mathrm{~mm}$ into the tibiofibular recess (arrow), suggestive of an interosseous ligament and membrane tear. F indicates fibula.
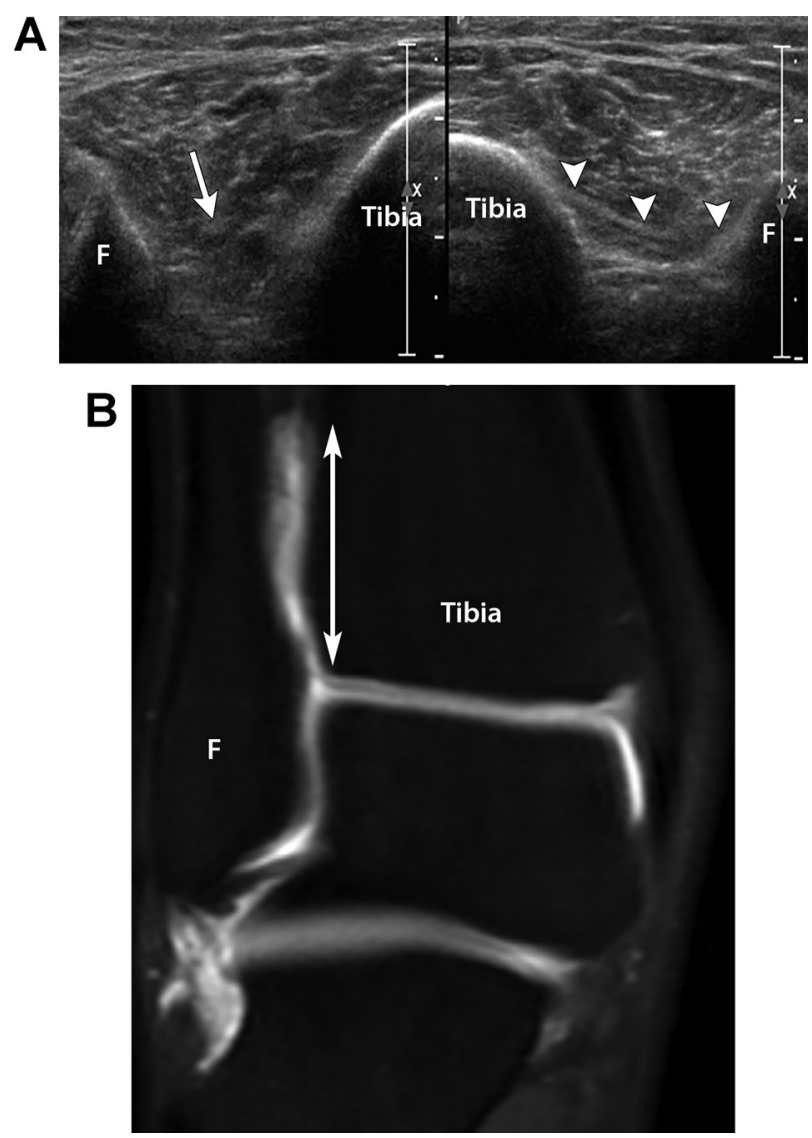

technique, and common pitfalls is important for the successful interpretation of ligamentous injuries of the ankle.

\section{References}

1. Khoury V, Guillin R, Dhanju J, Cardinal E. Ultrasound of ankle and foot: overuse and sports injuries. Semin Musculoskelet Radiol 2007; 11:149-161.

2. Peetrons P, Creteur V, Bacq C. Sonography of ankle ligaments. J Clin Ultrasound 2004; 32:491-499.
3. Bianchi S, Martinoli C, Gaignot C,De Gautard R, Meyer JM. Ultrasound of the ankle: anatomy of the tendons, bursae, and ligaments. Semin Musculoskelet Radiol 2005; 9:243-259.

4. Fessell DP,van Holsbeeck MT. Foot and ankle sonography. Radiol Clin North Am 1999; 37:831-858.

5. Patel S, Fessell DP, Jacobson JA, Hayes CW,van Holsbeeck MT. Artifacts, anatomic variants, and pitfalls in sonography of the foot and ankle. AJR Am J Roentgenol 2002; 178:1247-1254.

6. Fessell DP, Vanderschueren GM, Jacobson JA, et al. US of the ankle: technique, anatomy, and diagnosis of pathologic conditions. Radiographics 1998; 18:325-340.

7. Morvan G, Mathieu P, Busson J, Wybier M. Ultrasonography of tendons and ligaments of foot and ankle. J Radiol 2000; 81(suppl): 361-380.

8. Sconfienza LM, Orlandi D, Lacelli F, Serafini G, Silvestri E. Dynamic high-resolution US of ankle and midfoot ligaments: normal anatomic structure and imaging technique. Radiographics 2015; 35:164-178

9. Jacobson JA. Ankle, Foot, and Lower Leg Ultrasound. Fundamentals of Musculoskeletal Ultrasound. 2nd ed. Philadelphia, PA: Elsevier Saunders; 2013;212-256.

10. Morvan G, Busson J, Wybier M, Mathieu P. Ultrasound of the ankle. Eur J Ultrasound 2001; 14:73-82.

11. Choo HJ, Lee SJ, Kim DW, Jeong HW, Gwak H. Multibanded anterior talofibular ligaments in normal ankles and sprained ankles using 3D isotropic proton density-weighted fast spin-echo MRI sequence. AJR Am J Roentgenol 2014; 202:W87-W94.

12. Campbell DG, Menz A, Isaacs J. Dynamic ankle ultrasonography: a new imaging technique for acute ankle ligament injuries. Am J Sports Med 1994; 22:855-858.

13. Draghi F, Gregoli B, Bortolotto C. Absence of elevation of fibular tendons during dorsal hyperflexion of the foot: a sign of loss of the calcaneofibular ligament. J Ultrasound Med 2014; 33:1307-1308.

14. Oae K, Takao M, Uchio Y, Ochi M. Evaluation of anterior talofibular ligament injury with stress radiography, ultrasonography, and MR imaging. Skeletal Radiol 2010; 39:41-47.

15. Cheng Y, Cai Y, Wang Y. Value of ultrasonography for detecting chronic injury of the lateral ligaments of the ankle joint compared with ultrasonography findings. Br J Radiol 2014; 84:20130406.

16. Perrich KD, Goodwin DW, Hecht PJ, Cheung Y. Ankle ligaments on MRI: appearance of normal and injured ligaments. AJR Am J Roentgenol 2009; 193:687-695.

17. Campbell KJ, Michalski MP, Wilson KJ, et al. The ligament anatomy of the deltoid complex of the ankle: a qualitative and quantitative anatomical study. J Bone Joint Surg Am 2014; 96:e62.

18. Chhabra A, Subhawong TK, Carrino JA. MR imaging of deltoid ligament pathologic findings and associated impingement syndromes. Radiographics 2010; 30:751-761.

19. Kelikian SA. Anatomy of the Foot and Ankle: Descriptive, Topographic, Functional. 2nd ed. Philadelphia, PA: JB Lippincott Co; 1993. 
20. Crim J, Longenecker LG. MRI and surgical findings in deltoid ligament tears. AJR Am J Roentgenol 2015; 204:W63-W69.

21. Nazarenko A, Beltran LS, Bencardino JT. Imaging evaluation of traumatic ligamentous injuries of the ankle and foot. Radiol Clin North Am 2013; 51:455-478.

22. Stoller DW. Magnetic Resonance Imaging in Orthopaedics and Sports Medicine. 3rd ed. Philadelphia, PA: Lippincott Williams \& Wilkins; 2006.

23. Lechner R, Richter H, Friemert B, Palm HG, Gottschalk A. The value of ultrasonography compared with magnetic resonance imaging in the diagnosis of deltoid ligament injuries: is there a difference? Z Orthop Unfall 2015; 153:408-414.

24. Henari S, Banks LN, Radovanovic I, Queally J, Morris S. Ultrasonography as a diagnostic tool in assessing deltoid ligament injury in supination external rotation fractures of the ankle. Orthopedics 2011; 34:e639-e643.

25. Harish S, Jan E, Finlay K, et al. Sonography of the superomedial part of the spring ligament complex of the foot: a study of cadavers and asymptomatic volunteers. Skeletal Radiol 2007; 36:221-228.

26. Toye LR, Helms CA, Hoffman BD, Easley M, Nunley JA. MRI of spring ligament tears. AJR Am J Roentgenol 2005; 184:1475-1480.

27. Mansour R, Teh J, Sharp RJ, Ostlere S. Ultrasound assessment of the spring ligament complex. Eur Radiol 2008; 18:2670-2675.

28. Harish S, Kumbhare D, O’Neill J, Popowich T. Comparison of sonography and magnetic resonance imaging for spring ligament abnormalities: preliminary study. J Ultrasound Med 2008; 27: $1145-1152$

29. Durkee NJ, Jacobson JA, Jamadar DA, Femino JE, Karunakar MA, Hayes CW. Sonographic evaluation of lower extremity interosseous membrane injuries: retrospective review in 3 patients. J Ultrasound Med 2003; 22:1369-1375.

30. Hermans JJ, Beumer A,de Jong TA, Kleinrensink GJ. Anatomy of the distal tibiofibular syndesmosis in adults: a pictorial essay with a multimodality approach. J Anat 2010; 217:633-645.

31. Boonthathip M, Chen L, Trudell DJ, Resnick DL. Tibiofibular syndesmotic ligaments: MR arthrography in cadavers with anatomic correlation. Radiology 2010; 254:827-836.

32. Subhas N, Vinson EN, Cothran RL, Santangelo JR, Nunley JA II, Helms CA. MRI appearance of surgically proven abnormal accessory anterior-inferior tibiofibular ligament (Bassett's ligament). Skeletal Radiol 2008; 37:27-33.

33. Milz P, Milz S, Steinborn M, Mittlmeier T, Reiser M. 13-MHz high frequency ultrasound of the lateral ligaments of the ankle joint and the anterior tibia-fibular ligament: comparison and results of MRI in 64 patients. Radiologe 1999; 39:34-40.

34. Mei-Dan O, Kots E, Barchilon V, Massarwe S, Nyska M, Mann G. A dynamic ultrasound examination for the diagnosis of ankle syndesmotic injury in professional athletes: a preliminary study. Am J Sports Med 2009; 37:1009-1016. 